\title{
Coffee consumption and health: umbrella review of meta-analyses of multiple health outcomes
}

\author{
(C) $(\mathcal{\Theta}$ OPEN ACCESS
}

Coffee consumption and health: umbrella review of meta-analyses of multiple health outcomes In this paper by Poole and colleagues (BMJ 2017;359:j5024, doi:10.1136/bmj. j5024) an error spotted in a confidence interval led the authors to re-check their data. In doing so, they spotted a few other numerical errors. These do not affect their findings. In the estimate for all cause mortality in the abstract, the relative risk should read 0.83 (95\% confidence interval 0.79 to 0.88 ) rather than the current 0.83 ( 0.83 to 0.88 ). This error is repeated in the results section in the main text in the paragraph headed All cause mortality. In the same paragraph, the benefit at three decaffeinated cups a day should read $(0.89,0.85$ to 0.93$)$ rather than (0.83. 0.85 to 0.89$)$.

In figure 3, for any versus no coffee consumption, the estimate for liver cirrhosis is from the original paper (three cohorts and four case-control) rather than the reanalysis that the authors conducted on the cohort studies. The reanalysed estimate should be 0.89 ( 0.73 to 1.08$)$, rather than 0.61 (0.45 to 0.84 ) as shown. This would take it out of the top 10 most beneficial associations. This also applies to the estimate cited in the main text results section under the subheading Liver and gastrointestinal outcomes. In figure 4, for one extra cup a day, the estimate for cirrhosis has been converted by taking the cube root (for a three extra cups/day estimate) rather than the square root (for a two extra cup/day estimate). Consequently, it should read 0.77 (0.64 to 0.87 ) rather than 0.83 ( 0.78 to 0.88 ). This would not change its ranked position. In the results for Cardiovascular disease, mortality from stroke at three cups a day should read $(0.70,0.57$ to 0.86$)$ rather than $(0.70,0.80$ to 0.90$)$.

In the third paragraph of results for Cancer, the estimates for lung cancer for high versus low consumption are from the original paper rather than the authors' re-analysis. This should read odds ratio $(1.56,95 \%$ confidence interval 1.12 to 2.17 ), rather than $(1.59,95 \%$ confidence interval 1.26 to 2.00$)$. In the fourth paragraph of the Discussion, the figure for risk of fracture in women associated with one extra cup a day should read 5\% rather than $0.6 \%$. Also in the Strengths and weakness section of the discussion, for the $7 \%$ lower risk of all cause mortality in women the hazard ratio should read $(0.93,0.87$ to 0.98$)$ rather than $(0.93,0.82$ to 0.95$)$.

These changes also affected the web supplements and revised appendices 1 and 2 have been uploaded on bmj.com. 